\title{
Assessment of the reliability of real time ultrasound scanning to measure the humeral head position in a number of glenohumeral joint positions
}

\author{
Alya H. Bdaiwi", Lee Herrington, Adel Almangoush, Tanya Anne Mackenzie and Stuart B Porter \\ *Correspondence: a.h.bdaiwi@edu.salford.ac.uk

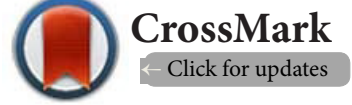 \\ College of Health, Sport and Rehabilitation Sciences, University of Salford, Salford M6 6PU, United Kingdom.
}

\begin{abstract}
The aim of the current study is to assess the intra-rater and inter-rater reliability of real time ultrasound scanning in measuring static humeral head position. Real time ultrasound scanning, an experimental clinical measurement, was used to record measurements of the humeral head position in a sample of (20) healthy volunteers [ 9 male, 11 female]. While the participant was seated in a standardized chair, Hips and knees were positioned at 90 degrees of flexion. The humeral head position of each subject was imaged at three ultrasound view [anterior, posterior and superior] in a different arm positions; images were repeated three times for each position by the same examiner on the same day to assess Intra-rater reliability. The Images were then analysed by the researcher and a second investigator to assess inter-tester reliability. Intra-rater and inter-rater reliability were quantified by using the intraclass correlation coefficient and standard error of measurement, the smallest detectable difference values were calculated and were used to estimate the magnitude of change that is predictable to exceed measurement error. The intra-rater reliability for all positions was found to be excellent for all tests ranged from 0.83 to 0.99 respectively. Inter -rater reliability between examiners was found to be good to excellent for all test positions ranged from 0.66 to 0.98 , and the standard error of measurement for all positions was less than the calculated mean. This study demonstrates that real time ultrasound scanning is a reliable method of assessing the location of the humeral head in a number of arm positions in healthy subjects when measured by the same examiner and this may suggest that RTUS could be used to assess the impact of rehabilitation programme or surgical interventions for shoulder pathology.
\end{abstract}

Keywords: Sonographic, repeatability, consistency, glenohumeral joint, shoulder impingement syndrome, shoulder abduction, distance measurement

\section{Introduction}

Shoulder disorders are the third most common musculoskeletal cause for consultation in primary care [1]. Physiotherapists managing these patients rely on accurate palpation and mobilization techniques to assist in the diagnosis and treatment of individuals with shoulder disorders. However, one of the limitations of palpation and mobilization techniques is that they may not provide quantified objective measurements of the shoulder [2]. There are now increasing moves towards the use of real time ultrasound scanning [RTUS] for investigating shoulder pathology due to its ability to provide a dynamic examination with high diagnostic accuracy [3] and the fact that it offers a potential method to visualize muscle contraction during examination and treatment [4].

RTUS has clinical uses in both static and dynamic measurement in a variety of positional assessments of the glenohumeral joint [5]. However; an important initial step in developing the use of RTUS further is to evaluate its reliability. As such, a reliable method of measurement could be used to assess the impact of rehabilitation or an intervention program for shoulder syndrome due to the liability of the measurement relating to the degree to which it is consistent and free from error [6].
Published studies [7-10] have investigated the reliability of clinically available methods to measure and evaluate the habitual humeral head position in relation to scapular landmarks to help in diagnosis and treatment of shoulder pathology. These studies all have potential limitations in the way the humeral head position was measured: they lack sufficient rigor in their measurement properties, such as imprecise description of landmarks and transducer placement [7] and small sample sizes [8-10]. This suggests that further investigation is necessary, as to date; there have been no protocols which have been developed to evaluate the reliability of RTUS scanning to measure glenohumeral joint position and function. Therefore, the objective of the present study was to assess intra- and inter-tester reliability of real time ultrasound to measure static humeral head position.

\section{Materials and method \\ Power analysis}

The Post-hoc power analysis calculations by Stratford and Goldsmith ${ }^{11}$,estimated that twelve subjects are required when three measurements are performed on each subject with a significance level of 0.05 and statistical power of 0.9. 
Bdaiwi et al. Physical Therapy and Rehabilitation 2014,

http://www.hoajonline.com/journals/pdf/2055-2386-1-1.pdf

In this study a power analysis was carried out using G Power software (version 3.1.7; Heinrich Heine University, Dusseldorf, Germany), with an effect size of 0.5 , a significance level of 0.05 , and a statistical power of 0.8 , the required sample size was calculated to be fifteen subjects. The sample size in this study was 20 participants.

\section{Participants}

A convenience sample of twenty healthy volunteers, nine males and eleven females, between the ages of 20-60 years, with a mean age of 33.4(SD=9.5), was recruited through verbal contact with people in the community and university students. All participants were right hand dominant.

Each subject was asked to read and sign a consent form. The eligibility requirement for inclusion in this study was that participants must be healthy subjects with no current or recurrent history of shoulder pain or surgery; subjects who had any shoulder or elbow pain in the previous six months before testing were excluded. The study was conducted at the School of Health, Sport \& Rehabilitation Sciences. Ethical approval was granted by the University of Salford Research Ethics Committee.

\section{Apparatus \& operator}

A diagnostic ultrasound imaging system Mylab 60 Esaote, Xvisoin model, with a 523 linear transducer and the frequency of the image set at $13 \mathrm{MHz}$ was used for scanning and recording the distance measurement. Before the commencement of the data collection processes, the devices were tested and calibrated.

\section{Procedure}

To standardize positioning, each subject was asked to sit with their shoulders exposed, on a customized chair with a short back support. Hips and knees were positioned at 90 degrees of flexion. The seated position was chosen to provide comfort to the subjects and to increase stability, as well as reducing the effect of fatigue.

Prior to each image capture, the arm was positioned appropriately by the examiner. An adjustable table and wedges were used to support the arm during the testing and a goniometer was used to ensure correct arm position before each image capture. In order to accurately place the probe, the examiner palpated and identified the following anatomical landmarks before each image capture:

1. The anterior head of the humerus and the coracoid process.

2. The posterior head of the humerus and the spine of the scapular.

3. The superior head of the humerus and the acromion process.

After identifying the necessary landmarks, the examiner standardised the ultrasound probe respectively on the anterior, posterior and superior aspects of the shoulder to capture images. The views on the screen were aligned and manually adjusted to capture the following hyper echoic landmarks:

1. The most anterior aspect of the humerus and coracoid process.

2. The most posterior aspect of the humerus and posterior glenoid.

3. The most superior aspect of the humerus and acromion process.

Images were captured in one session by one rater to assess test-retest reliability, and image analyses were done by two raters to assess inter-tester reliability of image analysis. The anterior and posterior views were captured with the arm in the following positions, also illustrated in (Figure 1) (in all instances the elbow was flexed to 90 degrees of flexion and the forearm rested in pronation on the support used):

1. Position one: shoulder neutral, with the arm at the side.

2. Position two: shoulder neutral, with the arm adjusted to incorporate 40 degrees external glenohumeral rotation.

3. Position three: 90 degrees of shoulder abduction with

0 degrees of glenohumeral external rotation.

4. Position four: 90 degrees shoulder abduction with 60 degrees of internal rotation.

5. Position five: 90 degrees shoulder abduction with 60 degrees of external rotation.

Ultrasound measurement of the superior view, or acro-miohumeral distance [AHD], was captured in two shoulder positions: neutral and 60 degrees of abduction. In both positions the

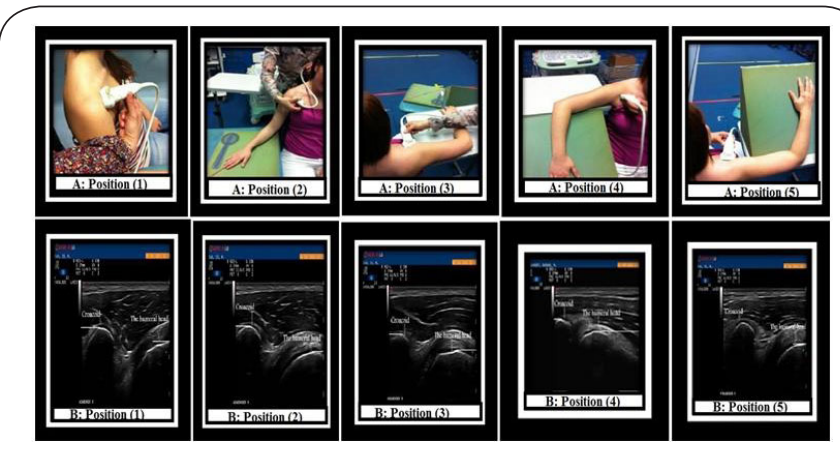

Figure 1. Test position for humeral head anterior imaging and ultrasound images capture of the most anterior aspect of the humerus and coracoid process.

A: An adjustable table and wedges were used to support the arm during the testing position. position (1) at rest, position (2) during 40 external rotation, position (3) during 90 abduction, position (4) during 90 abduction 60 external rotation, and position (5) 90 abduction 60 internal rotation. B: position (1) at rest, position (2) during 40 external rotation, position (3) during 90 abduction, position (4) during 90 abduction 60 external rotation, and position (5) 90 abduction 60 internal rotation. The humeral head and the borders of the coracoid are easily visible.

elbow was flexed to 90 degrees of flexion and the forearm rested in pronation on the support used. In this study, shoulder neutral and 60 degrees of shoulder abduction were chosen for the acromiohumeral measurement, because it is 
clinically noted that the painful arc, which may indicate a disorder of the subacromial region, is reported to start at 60 degrees of abduction [12]. In addition, RTUS imaging of the acromiohumeral distance is reported by previous authors to be less reliable at higher degrees of shoulder abduction [5].

During the testing, the order of testing was randomized, however, the position order adhered to the test conditions (always position 1 first followed by position 2 ...etc.).

\section{Measurement protocol}

The protocol of the ultrasound measurement was designed by a physiotherapist experienced in musculoskeletal scanning. All imaging was performed by one investigator, who attended ultrasound training and practiced the protocol under supervision prior to the study. Images were taken on both shoulders on the same day. Three consecutive ultrasound images were captured for each RTUS view, in each arm position, on each shoulder, resulting in seventy-two images per participant. All ultrasound images were saved onto an external hard drive from the ultrasound scanner for later measurement using Image J software. Images were then analyzed by the researcher and a second investigator to assess inter-tester reliability. Both investigators were blinded to each other's scores to avoid potential bias.

\section{Analysis of humeral head position Anterior ultrasound view}

As illustrated in (Figure 1), the transducer was placed transversely to capture the distance between the anterior humeral head and the coracoid. On the captured image, measurements were taken from the most anterior aspect of the humeral head to the most anterior aspect of the coracoid for each of the five arm positions.

\section{Posterior ultrasound view}

As illustrated in (Figure 2), the transducer was placed transversely at the level of the spinous process of the scapular to capture the distance between the posterior aspect of the humeral head and the most posterior rim of the glenoid. On the captured image, measurements were taken between these hyperechoic landmarks of the posterior aspect of the humeral head and the most posterior rim of the glenoid for each of the five arm positions.

\section{Superior ultrasound view}

As illustrated in (Figure 3), the transducer was placed on the lateral aspect of the acromion in line with the longitudinal axis of the humerus. On images, measurements were taken from the lateral edge of the acromion process of the scapula to the nearest margin of the humerus, with the arm in the two positions used for this view.

\section{Data processing}

Intra-class correlation [ICC] was used to calculate the reliability

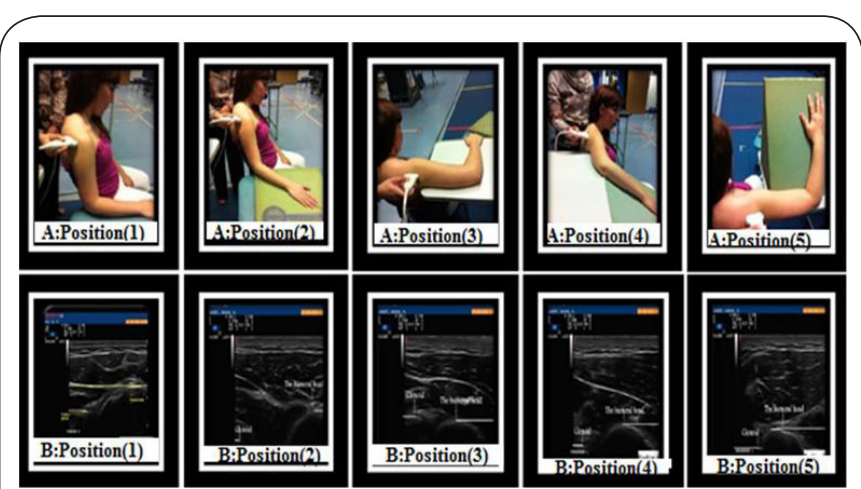

Figure 2. Test position for humeral head posterior imaging and ultrasound images capture of the most posterior aspect of the humerus and posterior glenoid.

A: An adjustable table and wedges were used to support the arm during the testing position: position (1) at rest, position (2) during 40 external rotation, position (3) during 90 abduction, position (4) during 90 abduction 60 external rotation, and position (5) 90 abduction 60 internal rotation. B: Position (1) at rest, position (2) during 40 external rotation, position (3) during 90 abduction, position (4) during 90 abduction 60 external rotation, and position (5) 90 abduction 60 internal rotation. The humeral head and the borders of the glenoid are easily visible.

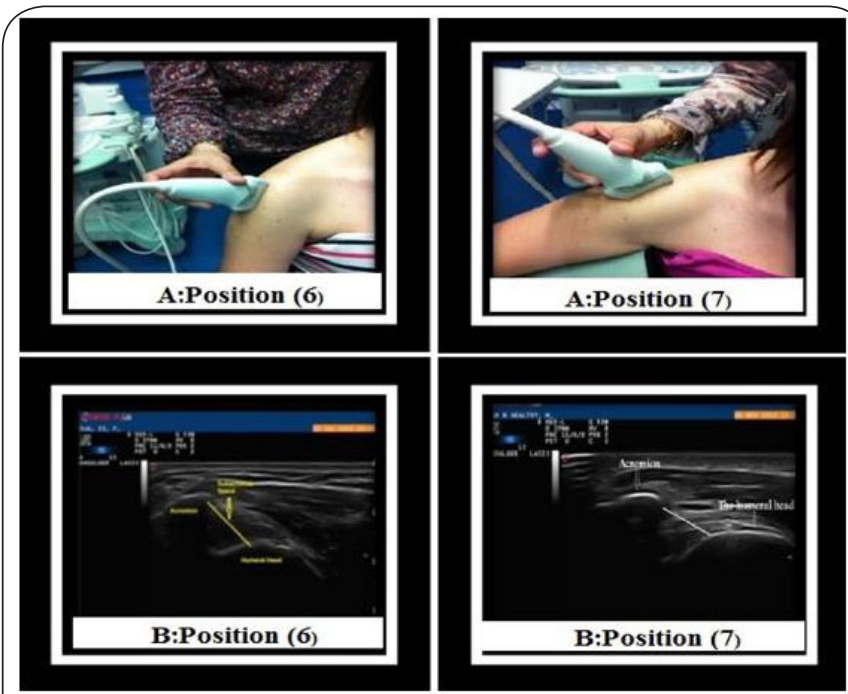

Figure 3. Test position for humeral head superior imaging and ultrasound images capture of the most superior aspect of the humerus and acromion process.

A: An adjustable table and wedges were used to support the arm during the testing position: position (6) at rest, position (7) during 60 degree abduction.

B: Position (6) at rest, position (7) during 60 degree abduction.

of the RTUS to measure the static humeral head position and acromiohumeral distance with a 95\% confidence interval using the Statistical Package for Social Sciences [SPSS] for Windows [Version 20.0, IBM SPSS]. Reliability was interpreted according to the ICC values: an ICC value of greater than or equal 
Bdaiwi et al. Physical Therapy and Rehabilitation 2014,

to 0.75 was considered excellent; if the value was between 0.4 to 0.75 it was considered good; a value less than 0.4 was deemed poor [13].

The interclass correlation coefficients values derived from single and averaged scores evaluated using the 2-way approach, coinciding with models interclass correlation coefficients 3,1 and $3, k$, respectively [15] (ICC3, 1) model was used for within-day intra-rater reliability and was based on a mixedeffect model with the mean of three trials from a single rater. Same-day inter-rater reliability was calculated using the ICC $(3, k)$ model, and was based on a random effects model with the second value indicating the number of raters performing the measurements ${ }^{14}$. In addition, in conjunction with ICC, the Standard Error of Measurement [SEM] was calculated to provide a range from the experimental score within which the true score of a measure is likely to lie [16]. The SEM was calculated using the formula: SEM $=S D \times(\sqrt{ } 1-I C C)$ Where SD is the standard deviation [17]. Moreover, the smallest detectable difference (SDD) has been obtained to allow determination of the change needed to indicate statistical significance [17]. The following formula was used to calculate SDD: SDD $=1.96 \times \sqrt{ } 2 \times S_{E M}{ }^{2}$. Independent t-tests were carried out to assess differences between dominant and non-dominant shoulders.

\section{Results}

All subjects completed all evaluations: therefore the analysis contains no drop-outs or missing data. Statistical testing to establish reliability and inferential statistics were done on all of the measurements collected from anterior, posterior and superior images in all arm positions.

There was no systematic difference between measurements for the dominant and non-dominant side in anterior, posterior or superior ultrasound views in all the various arm positions, as seen in (Table 1).

\section{Intra-tester reliability}

Intra-tester reliability was found to be excellent for all tests and is presented in (Table 2). ICCs ranged from 0.83 to 0.99 . SEM scores ranged from 0.5 to $1.3 \mathrm{~mm}$, this suggests that very little measurement error was evident.

\section{Inter-tester reliability}

Inter-tester reliability was found to be good to excellent for all tests and is presented in (Table 3). ICCs ranged from 0.66 to 0.98 . SEM scores ranged from 0.64 to 1.9 , suggesting that reliability between testers was good and very little measurement error was evident.

\section{Discussion}

The principal aim of this study was to assess intra- and interrater reliability of RTUS measurement of the humeral head position in a variety of arm positions in healthy subjects. It is necessary to determine whether the measurement really represents what is being measured: therefore, evaluating the
Table 1. Dominant and non-dominant comparison of averaged measured distances for all image views in all arm positions.

\begin{tabular}{llcll}
\hline Ultrasound views & Position & Men & Std.deviation & Sig.(2-tailed) \\
\hline Anterior distance & 1 & $(\mathrm{~mm})$ & $(\mathrm{mm})$ & \\
\hline & 2 & 0.8 & $( \pm 4.52)$ & 0.436 \\
& 3 & -1.4 & $( \pm 4.02)$ & 0.110 \\
& 4 & 0.4 & $( \pm 4.90)$ & 0.738 \\
& 5 & -0.3 & $( \pm 2.60)$ & 0.554 \\
\hline Posterior distance & 1 & 0.7 & $( \pm 4.38)$ & 0.453 \\
& 2 & 1.0 & $( \pm 4.85)$ & 0.342 \\
& 3 & -1.8 & $( \pm 5.52)$ & 0.140 \\
& 4 & -0.3 & $( \pm 5.95)$ & 0.840 \\
& 5 & 1.3 & $( \pm 6.69)$ & 0.370 \\
\hline Superior distance & 6 & 0.4 & $( \pm 2.86)$ & 0.505 \\
& 7 & -0.6 & $( \pm 2.59)$ & 0.280 \\
\hline
\end{tabular}

reliability of the ultrasound is important, since such reliability indicates the extent to which scores for a subject sample can be reproduced in the same participants in subsequent tests [6]. In the main, ICC values reflected good to excellent reliability, suggesting that the use of RTUS to determine the resting position in the humeral head in varying arm positions is reliable.

Knowledge of intra-tester reliability allows for a better understanding about the source of error of a measurement. The random error associated with a measure can be reduced if the experimenter's measures are consistent. Intra-tester reliability was found to be excellent for image capture in all positions and ranged from 0.83 to 0.99 . SEM scores ranged from 0.5 to 1.3 $\mathrm{mm}$, suggesting that very little measurement error was evident. The low SEM values show that there is minimal contribution of experimenter error to the overall error of the measure and error is due to systematic bias or other within-subject variation. In addition to the excellent intra-tester reliability scores demonstrated, inter-tester reliability was also found to be good to excellent, with ICCs ranging from 0.66 to 0.98 , with low associated SEM values. SEM scores ranged from 0.64 to 1.90 , suggesting that reliability between testers was good and very little measurement error was evident. Therefore, we can be confident that the measure is stable between different examiners.

Anterior ultrasound view: Previous studies that measured the anterior aspect resulted in poor image quality, leading to incomparable measurements. For instance, the study by Krarup et al., [18,19] failed to provide sufficient penetration to adequately define the anterior glenoid distance measurement due to the investigators using a $3.5 \mathrm{MHz}$ curved array transducer, resulting in alteration and curving of the images; this may have led to incorrect determination of the landmark [5]. However, 
Table 2. Intratester reliability. Mean standard deviation (SD), intraclass correlation coefficient (ICC), $95 \%$ confidence intervals (CI) for ICC, standard error of measurement (SEM), and smallest detectable difference (SDD) values for the three ultrasound views for dominant and non-dominant shoulders.

\begin{tabular}{|c|c|c|c|c|c|c|c|c|c|c|c|c|c|}
\hline \multirow{3}{*}{$\begin{array}{l}\text { Ultrasound } \\
\text { view }\end{array}$} & \multirow{3}{*}{ position } & \multicolumn{6}{|c|}{ Dominant } & \multicolumn{6}{|c|}{ Non-Dominant } \\
\hline & & \multirow{2}{*}{$\begin{array}{l}\text { Mean SD } \\
(\mathrm{mm})\end{array}$} & \multirow[t]{2}{*}{ ICC } & \multicolumn{2}{|c|}{$95 \%$ IC } & \multirow{2}{*}{$\begin{array}{l}\text { SEM } \\
(\mathrm{mm})\end{array}$} & \multirow{2}{*}{$\begin{array}{l}\text { SDD } \\
(\mathrm{mm})\end{array}$} & \multirow{2}{*}{$\begin{array}{l}\text { MEAN } \\
\text { SD } \\
(\mathrm{mm}) \\
\end{array}$} & \multirow[t]{2}{*}{ ICC } & \multicolumn{2}{|c|}{$95 \%$ IC } & \multirow{2}{*}{$\begin{array}{l}\text { SEM } \\
(\mathrm{mm})\end{array}$} & \multirow{2}{*}{$\begin{array}{l}\text { SDD } \\
(\mathrm{mm}) \\
\end{array}$} \\
\hline & & & & Lower & upper & & & & & Lower & upper & & \\
\hline \multirow[t]{5}{*}{ Anterior } & 1 & $2.6(4.5)$ & 0.91 & 0.83 & 0.96 & 1.3 & 3.7 & $1.2(4.5)$ & 0.97 & 0.92 & 0.98 & 0.7 & 2.1 \\
\hline & 2 & $3.5(3.7)$ & 0.93 & 0.85 & 0.96 & 1.0 & 2.7 & $4.3(4.1)$ & 0.98 & 0.96 & 0.99 & 0.5 & 1.6 \\
\hline & 3 & $3.1(2.9)$ & 0.89 & 0.78 & 0.95 & 0.98 & 2.7 & $1.5(4.0)$ & 0.95 & 0.89 & 0.97 & 0.9 & 2.5 \\
\hline & 4 & $1.7(4.1)$ & 0.93 & 0.86 & 0.96 & 1.1 & 3.0 & $2.0(3.8)$ & 0.97 & 0.94 & 0.98 & 0.67 & 1.8 \\
\hline & 5 & $4.7(3.8)$ & 0.96 & 0.92 & 0.98 & 0.77 & 2.1 & $4.4(3.3)$ & 0.95 & 0.89 & 0.97 & 0.74 & 2.0 \\
\hline \multirow[t]{5}{*}{ Posterior } & 1 & $7.3(3.3)$ & 0.96 & 0.92 & 0.98 & 0.66 & 1.8 & $6.8(3.6)$ & 0.95 & 0.90 & 0.97 & 0.81 & 2.3 \\
\hline & 2 & $9.9(3.1)$ & 0.92 & 0.83 & 0.96 & 0.90 & 2.5 & $8.9(4.3)$ & 0.93 & 0.86 & 0.97 & 1.1 & 3.1 \\
\hline & 3 & $2.6(3.5)$ & 0.90 & 0.81 & 0.95 & 1.1 & 3.1 & $4.4(4.9)$ & 0.97 & 0.94 & 0.98 & 0.86 & 2.3 \\
\hline & 4 & $3.5(4.4)$ & 0.93 & 0.86 & 0.96 & 1.2 & 3.2 & $3.8(3.2)$ & 0.96 & 0.92 & 0.98 & 0.65 & 1.8 \\
\hline & 5 & $4.6(4.3)$ & 0.98 & 0.95 & 0.99 & 0.62 & 1.7 & $3.2(6.3)$ & 0.98 & 0.97 & 0.99 & 0.90 & 2.5 \\
\hline \multirow{2}{*}{$\begin{array}{l}\text { Superior } \\
\text { [AHD] }\end{array}$} & 6 & $8.3(1.9)$ & 0.83 & 0.69 & 0.92 & 0.78 & 2.1 & $8.6(2.2)$ & 0.86 & 0.74 & 0.93 & 0.82 & 2.2 \\
\hline & 7 & 7.8(1.7) & 0.83 & 0.68 & 0.92 & 0.19 & 1.7 & $7.3(2.3)$ & 0.88 & 0.77 & 0.94 & 0.78 & 2.1 \\
\hline
\end{tabular}

Table 3 - Inter-tester reliability. Mean standard deviation (SD), interclass correlation coefficient (ICC), 95\% confidence intervals (CI) for ICC, standard error of measurement (SEM), and smallest detectable difference (SDD) values for the three ultrasound views for dominant and non-dominant shoulder.

\begin{tabular}{|c|c|c|c|c|c|c|c|c|c|c|c|c|c|}
\hline \multirow{3}{*}{$\begin{array}{l}\text { Ultrasound } \\
\text { view }\end{array}$} & \multirow{3}{*}{ position } & \multicolumn{6}{|c|}{ Dominant } & \multicolumn{6}{|c|}{ Non-Dominant } \\
\hline & & Mean SD & ICC & $95 \%$ IC & & SEM & SDD & MEAN SD & ICC & $95 \%$ IC & & SEM & SDD \\
\hline & & $(\mathrm{mm})$ & & Lower & upper & $(\mathrm{mm})$ & $(\mathrm{mm})$ & $(\mathrm{mm})$ & & Lower & upper & $(\mathrm{mm})$ & $(\mathrm{mm})$ \\
\hline \multirow[t]{5}{*}{ Anterior } & 1 & $3.9(2.2)$ & 0.85 & 0.62 & 0.93 & 0.85 & 2.3 & $3.1(2.8)$ & 0.95 & 0.87 & 0.98 & 0.64 & 1.7 \\
\hline & 2 & $3.6(2.7)$ & 0.70 & 0.24 & 0.88 & 1.5 & 4.2 & $5.6(3.5)$ & 0.69 & 0.25 & 0.87 & 1.9 & 5.5 \\
\hline & 3 & $3.4(2.1)$ & 0.76 & 0.40 & 0.90 & 1.0 & 2.9 & $3.3(2.9)$ & 0.66 & 0.16 & 0.86 & 1.6 & 4.7 \\
\hline & 4 & $3.4(2.8)$ & 0.87 & 0.67 & 0.95 & 0.82 & 2.9 & $3.7(2.3)$ & 0.78 & 0.43 & 0.91 & 1.1 & 3.0 \\
\hline & 5 & $4.5(3.4)$ & 0.80 & 0.51 & 0.92 & 1.5 & 4.2 & $4.4(2.9)$ & 0.72 & 0.30 & 0.89 & 1.5 & 4.3 \\
\hline \multirow[t]{5}{*}{ Posterior } & 1 & $7.3(2.8)$ & 0.71 & 0.30 & 0.88 & 1.5 & 4.2 & 7.1(3.5) & 0.71 & 0.30 & 0.88 & 1.8 & 5.4 \\
\hline & 2 & $10.2(2.6)$ & 0.77 & 0.42 & 0.91 & 1.2 & 3.5 & $9.5(4.1)$ & 0.81 & 0.54 & 0.92 & 1.8 & 5.0 \\
\hline & 3 & $2.6(2.4)$ & 0.74 & 0.35 & 0.89 & 1.2 & 3.4 & $4.7(3.9)$ & 0.84 & 0.60 & 0.93 & 1.5 & 4.3 \\
\hline & 4 & $3.5(3.6)$ & 0.87 & 0.67 & 0.95 & 1.3 & 3.6 & $3.7(2.5)$ & 0.73 & 0.35 & 0.89 & 1.3 & 3.6 \\
\hline & 5 & $4.8(4.8)$ & 0.85 & 0.63 & 0.94 & 1.1 & 3.0 & $6.8(4.4)$ & 0.77 & 0.33 & 0.91 & 2.1 & 5.8 \\
\hline \multirow{2}{*}{$\begin{array}{l}\text { Superior } \\
\text { [SAS] }\end{array}$} & 6 & $8.2(1.8)$ & 0.86 & 0.66 & 0.94 & 0.78 & 2.1 & $8.6(2.1)$ & 0.72 & 0.30 & 0.89 & 0.82 & 2.3 \\
\hline & 7 & $7.8(1.7)$ & 0.85 & 0.64 & 0.94 & 0.71 & 1.9 & $7.2(2.2)$ & 0.74 & 0.35 & 0.89 & 0.78 & 2.2 \\
\hline
\end{tabular}

the excellent reliability reported in this study may be due to accurate identification of the shoulder anatomical landmarks. It has been shown that well-defined anatomical landmarks contribute to high intra/inter-reliability [20].

Posterior ultrasound view: This study demonstrated excellent Intra-tester reliability and good to excellent Inter-tester reliability for the measurement of the posterior aspect in all positions used; this is in agreement with the study by Borsaet al., [10], which reported excellent inter-rater reliability.

The acromiohumeral distance [AHD] measurement in this study established excellent reliability for measurements taken in the neutral and 60 degrees shoulder abduction positions. This is in agreement with previous studies on healthy individuals $[2,7,9]$ that have reported that RTUS is a reliable method. However, 
Bdaiwi et al. Physical Therapy and Rehabilitation 2014,

the methodological and positional difference between studies limits more direct comparison of the results.

Previous studies have reported acromiohumeral distances, but used different modalities such as radiography, magnetic resonance imaging and in vitro measures. Ranges of AHD are reported between $6 \mathrm{~mm}$ and $16 \mathrm{~mm}$ in healthy individuals $[21,22]$. Studies using the RTUS method to measure this distance have recorded values ranging between $5.6 \mathrm{~mm}$ and $13.4 \mathrm{~mm}[6,23-28]$. These findings are similar to this study measurements, which ranged between $5 \mathrm{~mm}$ and $14 \mathrm{~mm}$.

Findings from this study provide evidence of the clinical usefulness of RTUS measurement of the humeral head position. Observation and measurement of the habitual anterior, posterior and superior static humeral head positions in relation to scapular landmarks may be important for investigating shoulder pathology and the impact of rehabilitation strategies and surgery on humeral head position. This technique has potential in the assessment of the acromiohumeral distance, since reduction in this distance is associated with underlying mechanical compression of the subacromial structures on the anterior under surface of the acromion and the head of the humerus, causing symptoms of subacromial impingement syndrome [29]. Laxity of the glenohumeral joint is also considered to be part of the pathological mechanics that contribute to internal impingement syndrome in the shoulder [10], making quantification of the anterior and posterior resting position of the humerus important.

No significant difference was found in the humeral head position between dominant and non-dominant shoulders, which would indicate that hand dominance in the normal population does not affect humeral head position.

The present study has two main limitations: firstly, the inter-tester reliability of image capture was not investigated; secondly, randomization of the order of image capture was not done. It must also be borne in mind that the sample population in this study was healthy and young; how this may reflect individuals who are undergoing injury rehabilitation or older age groups is unclear and further research should be conducted in this area.

\section{Conclusion}

In conclusion, intra-test reliability of capturing RTUS measurements of static humeral head position and acromiohumeral distance in healthy individuals within the same day was observed to be very reliable when assessed by one examiner, and intertester reliability between two examiners analysing images was good. It can be concluded that the RTUS is suitable for use in clinical practice and further research. Clinicians and researchers could use this method to collect normalised and raw score reference data to help evaluate whether changes in an individual's performance during rehabilitation is a true reflection of progress. This technique provides clinicians with a tool to identify changes in the humeral head position.

\section{List of abbreviations}

RTUS: Real time ultrasound scanning

AHD: Acromiohumeral distance

ICC: Intra-class correlation

SEM: Standard Error of Measurement

SD: Standard deviation

SDD: The smallest detectable difference

\section{Competing interests}

The authors declare that they have no competing interests.

Authors' contributions

\begin{tabular}{|l|c|c|c|c|c|}
\hline Authors' contributions & AHB & LH & AA & TAM & SBP \\
\hline Research concept and design & $\checkmark$ & $\checkmark$ & -- & -- & -- \\
\hline Collection and/or assembly of data & $\checkmark$ & $\checkmark$ & $\checkmark$ & $\checkmark$ & -- \\
\hline Data analysis and interpretation & $\checkmark$ & $\checkmark$ & -- & $\checkmark$ & -- \\
\hline Writing the article & $\checkmark$ & $\checkmark$ & $\checkmark$ & -- & $\checkmark$ \\
\hline Critical revision of the article & $\checkmark$ & $\checkmark$ & $\checkmark$ & $\checkmark$ & $\checkmark$ \\
\hline Final approval of article & $\checkmark$ & $\checkmark$ & $\checkmark$ & $\checkmark$ & $\checkmark$ \\
\hline Statistical analysis & $\checkmark$ & $\checkmark$ & $\checkmark$ & -- & -- \\
\hline
\end{tabular}

Acknowledgement

The authors would like to express our gratitude to the Ministry of Higher Education, Republic of Iraq, for funding this research, which was undertaken as part of a doctoral thesis.

\section{Publication history}

Editor: Anh-Dung Nguyen, High Point University, USA.

Received: 11-Dec-2013 Revised: 01-Feb-2014

Accepted: 12-Feb-2014 Published: 12-Mar-2014

\section{References}

1. Dinnes J, Loveman $E, M c I n t y r e ~ L$ and Waugh $N$. The effectiveness of diagnostic tests for the assessment of shoulder pain due to soft tissue disorders: a systematic review. Health Technol Assess. 2003; 7:iii, 1-166. | Article | PubMed

2. Kumar P, Chetwynd J, Evans A, Wardle G, Crick C and Richardson B. Interrater and intrarater reliability of ultrasonographic measurements of acromion-greater tuberosity distance in healthy people. Physiother Theory Pract. 2011; 27:172-5. | Article | PubMed

3. Lew HL, Chen CP, Wang TG and Chew KT. Introduction to musculoskeletal diagnostic ultrasound: examination of the upper limb. Am J Phys Med Rehabil. 2007; 86:310-21. | Article | PubMed

4. Talbott NR and Witt DW. Ultrasound imaging of the serratus anterior muscle at rest and during contraction. Clin Physiol Funct Imaging. 2013; 33:192-200. | Article | PubMed

5. Duerr M. Reliability and accuracy of distance measurements between shoulder bony landmarks evaluated by ultrasound in asymptomatic subjects. PhD diss., AUT University, 2010. | Article

6. McCreesh, Karen M, James M. Crotty, and Jeremy S. Lewis. Acromiohumeral distance measurement in rotator cuff tendinopathy: is there a reliable, clinically applicable method? A systematic review. British journal of sports medicine 2013. | Article

7. Pijls BG, Kok FP, Penning LI, Guldemond NA and Arens HJ. Reliability study of the sonographic measurement of the acromiohumeral distance in symptomatic patients. J Clin Ultrasound. 2010; 38:128-34. | Article | PubMed

8. Schmidt WA, Schmidt H, Schicke B and Gromnica-Ihle E. Standard reference values for musculoskeletal ultrasonography. Ann Rheum Dis. 2004; 63:988-94. | Article | PubMed Abstract | PubMed Full Text

9. Na A. D. Ultrasonographic Measurement of the Acromio-Humeral 
Bdaiwi al. Physical Therapy and Rehabilitation 2014,

Distance at the Inlet of the Subacromial Space: A Reliability Study. Clin J Sports Med. 2000; 10:221. | Article

10. Borsa PA, Scibek JS, Jacobson JA and Meister K. Sonographic stress measurement of glenohumeral joint laxity in collegiate swimmers and age-matched controls. Am J Sports Med. 2005; 33:1077-84. | Article | PubMed

11. Stratford PW and Goldsmith $\mathrm{CH}$. Use of the standard error as a reliability index of interest: an applied example using elbow flexor strength data. Phys Ther. 1997; 77:745-50. | Article | PubMed

12. Kessel $L$ and Watson $M$. The painful arc syndrome. Clinical classification as a guide to management. J Bone Joint Surg Br. 1977; 59:166-72. Article | PubMed

13. Rosner B.Fundamentals of biostatistics. Belmont, Calif Duxbury Press; 1995:345-442. | Pdf

14. Shrout PE and Fleiss JL. Intraclass correlations: uses in assessing rater reliability. Psychol Bull. 1979; 86:420-8. | Article | PubMed

15. Weir JP. Quantifying test-retest reliability using the intraclass correlation coefficient and the SEM. J Strength Cond Res. 2005; 19:23140. | Article | PubMed

16. Thomas $\mathrm{J}$ and Nelson JK. Research methods in physical activity. Champaign, IL: Human Kinetics; 2005. | Book

17. Atkinson $G$ and Nevill AM. Statistical methods for assessing measurement error (reliability) in variables relevant to sports medicine. Sports Med. 1998; 26:217-38. | Article | PubMed

18. Court-Payen M. Real-time sonography of anterior translation of the shoulder: An anterior approach. Eur J Ultrasound. 1995; 2:283-7. I Article

19. Krarup AL, Court-Payen M, Skjoldbye B and Lausten GS. Ultrasonic measurement of the anterior translation in the shoulder joint. J Shoulder Elbow Surg. 1999; 8:136-41. | Article | PubMed

20. Balint PV and Sturrock RD. Intraobserver repeatability and interobserver reproducibility in musculoskeletal ultrasound imaging measurements. Clin Exp Rheumatol. 2001; 19:89-92. | PubMed

21. Petersson $\mathrm{CJ}$ and Redlund-Johnell I. The subacromial space in normal shoulder radiographs. Acta Orthop Scand. 1984; 55:57-8. | Article | PubMed

22. Flatow EL, Soslowsky LJ, Ticker JB, Pawluk RJ, Hepler M, Ark J, Mow VC and Bigliani LU. Excursion of the rotator cuff under the acromion. Patterns of subacromial contact. Am J Sports Med. 1994; 22:779-88. Article | PubMed

23. Cheng SC, Hulse D, Fairbairn KJ, Clarke M and Wallace WA. Comparison of dynamic ultrasound and stress radiology for assessment of inferior glenohumeral laxity in asymptomatic shoulders. Skeletal Radiol. 2008; 37:161-8. | Article | PubMed

24. Cholewinski JJ, Kusz DJ, Wojciechowski P, Cielinski LS and Zoladz MP. Ultrasound measurement of rotator cuff thickness and acromiohumeral distance in the diagnosis of subacromial impingement syndrome of the shoulder. Knee Surg Sports Traumatol Arthrosc. 2008; 16:408-14. | Article | PubMed

25. Desmeules F, Minville L, Riederer B, Cote CH and Fremont P. Acromiohumeral distance variation measured by ultrasonography and its association with the outcome of rehabilitation for shoulder impingement syndrome. Clin J Sport Med. 2004; 14:197-205. | Article | PubMed

26. Jerosch J, Marquardt M and Winkelmann W. [Ultrasound documentation of translational movement of the shoulder joint. Normal values and pathologic findings]. Ultraschall Med. 1991; 12:31-5. | Article | PubMed

27. Silva RT, Hartmann LG, Laurino CF and Bilo JP. Clinical and ultrasonographic correlation between scapular dyskinesia and subacromial space measurement among junior elite tennis players. Br J Sports Med. 2010; 44:407-10. | Article | PubMed

28. Wang HK, Lin JJ, Pan SL and Wang TG. Sonographic evaluations in elite college baseball athletes. Scand J Med Sci Sports. 2005; 15:29-35. I Article I PubMed

29. Ludewig PM and Braman JP. Shoulder impingement: biomechanical considerations in rehabilitation. Man Ther. 2011; 16:33-9. | Article |
PubMed Abstract | PubMed Full Text

\section{Citation:}

Bdaiwi AH, Herrington L, Almangoush A, Mackenzie TA and Porter SB. Assessment of the reliability of real time ultrasound scanning to measure the humeral head position in a number of glenohumeral joint positions. Phys Ther Rehabil. 2014; 1:1. http://dx.doi.org/10.7243/2055-2386-1-1 